Editorial

\title{
Acknowledgement to Reviewers of Molecules in 2013
}

Molecules Editorial Office

MDPI AG, Klybeckstrasse 64, CH-4057 Basel, Switzerland

Published: 25 February 2014

The editors of Molecules would like to express their sincere gratitude to the following reviewers for assessing manuscripts in 2013:

Loboda, A.

Abaev, Vladimir

Abbotto, Abbotto

Abdel-Gawad, Fatma M.

Abdel-Megid, Mohamed

Abdelwhab, E. M.

Abdullah, Abdullah

Abonía, Rodrigo

Adam, Vojtech

Adiele, R. C.

Adireddy, Shiva

Afantitis, Antreas

Afonso, Carlos A. M.

Agarwal, Umesh P.

Agazzi, Agazzi

Agbor, Gabriel A.

Agbossou, Francine

Agrofoglio, Luigi

Ahmad, Ijaz

Ahmed, Mukhtar H.

Ahn, Joong-Hoon

Ahn, Sung-Hoon

Aires, Alfredo

Aisa, Haji Akber
Ait-Oudhia, Sihem

Akamatsu, Miki

Akhtar, Yasmin

Al-Tel, Taleb

Al. Marghitas, Liviu

Alam, M. S.

Alam, Todd

Albericio, Fernando

Albert, Joan

Alcântara, Ana C. S.

Alcaráz, Lucía Esther

Alché, Laura E.

Alebic-Kolbah, Tanja

Alemán, José

Antunes, Alexandra M. M.

Alezra, Valérie

Alhaique, Franco

Ali, Ihsan

Ali, Saqib

Alkharfy, Khalid M.

Allan, Andrew C.

Allison, Stuart A.

Almajano, Maria Pilar

Almeida, Wanda
Almendros, Pedro

Almerico, Anna Maria

Alonso, Diego A.

Alunda, José Ma

Alwan, Shakir Mahmood

Alway, Stephen E.

Amabilino, David B.

Amann, Anton

Amarowicz, Ryszard

Amat, Mercedes

Amboni, R. D. M. C.

Ambrosio, Sergio R.

Ambudkar, Suresh V.

Amin, Kawa

Anand, Sathanandam S.

Anderson, Carolyn

Anderson, J. P.

Andjelkovic, Anuska V.

Andrade, Carolina Horta

Andrade, Paula Branquinho

Andreana, Peter R.

Andreasen, Peter A.

Andrés, Maria Fe

Andrisano, Vincenza 
Angela, Bisio

Anibal, Paula

Anissimov, Yuri G.

Anna, Horszwald

Annaert, Pieter

Anraku, Makoto

Antipenko, Lyudmila N.

Antje, Huefner

Antonelli, Alessandro

Anzai, Jun-ichi

Apone, Fabio

Aponick, Aaron

Arai, Masayoshi

Arano, Yasushi

Archibald, Steve J.

Arct, Jacek

Arellano, Juan B.

Arenas, Miguel

Ariga, Katsuhiko

Arosio, Daniela

Arratia-Pérez, Ramiro

Arrieta, Jesús

Aruoma, Okezie

Asai, Akira

Asai, Teigo

Asea, Alexzander

Asís, Silvia E.

Assimopoulou, Andreana N.

Astakhova, I. Kira

Asti, Mattia

Ata, Athar

Atrian, Sílvia

Attioua, Barthelemy K.

Aullon, Gabriel

Avarvari, Narcis

Avato, Pinarosa

Avula, Bharathi

Ayala-Zavala, J. F.

Aydemir, Adnan

Ayyalusamy, Ramamoorthy

Azam, Faizul

$\mathrm{Ba}$, Yong
Baba, Akio

Babula, Petr

Bach, Horacio

Bachran, Christopher

Baczek, Tomasz

Baek, Hyung Hee

Baek, Nam In

Baeza, Alejandro

Bagnoli, Luana

Bahn, Andrew

Bai, Hanying

Bai, Meng-Yi

Baiano, A.

Baker, C. Jacyn

Baker, Michael E.

Bal, Wojciech

Balagiannis, Dimitrios

Baldwin, Elizabeth

Balemba, Onesmo B.

Ballatore, Carlo

Balling Engelsen, Søren

Baltina, L. A.

Baltora-Rosset, Sylvie

Balzarini, Jan

Bando, Yasuko

Banerjee, Sanjeev

Bang, Jeong Kyu

Banghart, Matthew

Bánóczi, Zoltán

Bansal, Ranju

Banskota, Arjun H.

Bao, Chongyun

Bao, Ming

Bao, Yongping

Baqi, Younis

Barbas, Carlos F.

Barbera, Mariagnese

Barbosa-Pereira, Letricia

Barboza, M.

Bardi, Giuseppe

Bardón, Alicia

Barker, David
Barluenga, José

Barone, Giampaolo

Barr, John

Barreira, João

Barreto Silva, Fátima Regina Mena

Barreto-Bergter, Eliana

Barreto, Maria Do Carmo

Barrio, Pablo

Bass, Chris

Bastos, J. K.

Batanero, Belen

Batista, Ronan

Batovska, Daniela

Battino, Maurizio

Baudequin, Christine

Bavaresco, Luigi

Bavetsias, Vassilios

Baxendale, Ian

Bayse, Craig A.

Beaucage, Serge

Bechthold, Andreas

Beck-Sickinger, Annette

Beck, Melinda A.

Bednarczyk-Cwynar, Barbara

Bekhit, Aladin

Bell, Stephen

Bellinger, Rick

Belmonte, Mark

Ben-Shabat, Shimon

Bencini, Andrea

Bencivenni, Giorgio

Benites, Julio

Benke, Dietmar

Benkendorff, Kirsten

Bennet, Andrew

Benoist, Eric

Berestetskii, Berestetskii

Bergamini, Paola

Berger, Walter

Berlin, Darrell

Bermúdez-Aguirre, Daniela

Bermúdez-Torres, Kalina 
Bernardi, Luca

Bernstein, Elliot R.

Bertelli, Davide

Berti, Debora

Bertolini, Joseph

Besson, Thierry

Best, Michael D.

Beutler, John A.

Bhat, Shridhar

Bhowmik, Prasanta

Bhushan, Alok

Bianchi, Antonio

Bielawski, Christopher W.

Biersack, Bernhard

Biesaga, Magdalena

Bijak, Michał

Bilsland, Elizabeth

Biot, Christophe

Birner-Gruenberger, Ruth

Biroccio, Annamaria

Bishayee, Anupam

Blaazer, Antoni R.

Blackburn, Michael

Blackstone, Neil W.

Blackwell, Helen E.

Blair, Richard G.

Blais, Anne

Blanot, Didier

Blažević, I.

Blindauer, Claudia A.

Boa, Andrew N.

Bobe, Gerd

Boersma, Hendrikus H.

Böhm, Volker

Bokoch, Michael P.

Bommareddy, Ajay

Bonifácio, Vasco

Boo, Yong Chool

Bordiga, Matteo

Bordoni, Alessandra

Borges, Rosivaldo

Boros, Laszlo
Borovkov, Nicholas Yu

Borrelli, Francesca

Borriello, Carmela

Botta, Mauro

Bouaziz, Serge

Bouet, Gilles

Boulanger, É.

Boulogne, Isabelle

Bouquillon, Sandrine

Bovell-Benjamin, A. C.

Brackman, Gilles

Bradshaw, Claire E.

Braissant, O.

Brandão, Maria das Graças

Brannon, Patsy M.

Braslavsky, Silvia E.

Brattström, A.

Braun, Klaus

Bravo, Héctor R.

Brazel, Christopher S.

Brazier, John A.

Breckenridge, Ross

Brecker, Lothar

Brehm, John

Breitling, Frank

Bremner, John B.

Brinker, Thomas

Broderick, Patricia A.

Brown, Andrew

Brown, Dan

Brown, Lindsay

Brown, Richard C. D.

Brudzynski, Katrina

Bruheim, Skjalg

Brüning, Ansgar

Brunner, Eike

Bruno, Maurizio

Brus, Jiř́i

Bruschi, Marcos

Brüschweiler, Rafael

Brusotti, G.

Brust, Peter
Bruzik, Karol

Bryant-Friedrich, Amanda

Bryant, Joseph

Bryce, David L.

Brycki, Bogumił

Brzezinski, Krzysztof

$\mathrm{Bu}$, Xianhe

$\mathrm{Bu}$, Xianzhang

Buchbauer, Gerhard

Büchel, Claudia

Budzisz, Elzbieta

Bühler, Bruno

Bujoli, Bruno

Bukowska, Bożena

Bundle, David R.

Buolamwini, John K.

Burger, Alain

Burghardt, Nesha S.

Burketova, L

Burks, Charles S.

Burt, Sa

Bustamante, J.

Bustos, Daniel A.

Buszek, Keith R.

Butin, Alexander V.

Butler, Alison

Byk, Gerardo

Caballero, Julio

Caboni, Pierluigi

Cabrera, Carmen

Cabrera, Margarita Gutiérrez

Čačić, Milan

Cadierno, Victorio

Cadwallader, Keith

Cai, Jianfeng

Cai, Lisheng

Cai, Shaoqing

Calderon, Angela

Caliandro, Rocco

Calín-Sánchez, Ángel

Calmès, Monique

Camacho-Corona, M. R. 


\begin{tabular}{|c|c|c|}
\hline Camara, Bilal & Castro, Bruno M. & Chen, Ji Jun \\
\hline Câmara, José & Castro, Helena Carla & Chen, Jiangshan \\
\hline Camargo, Carlos & Castro, Solange & Chen, Jianhan \\
\hline Camins, Antoni & Casu, M. Benedetta & Chen, Jianjun \\
\hline Campidelli, Stéphane & Cavar, Sanja & Chen, Jih-Jung \\
\hline Campiglia, Pietro & Cavazza, Antonella & Chen, Kai \\
\hline Campos, Carmen A. & Cavicchi, Kevin & Chen, Menglin \\
\hline Campos, Joaquín M. & Cawkwell, Marc & Chen, Nai-Hong \\
\hline Canac, Yves & Celzard, Alain & Chen, Oliver \\
\hline Candeias, Nuno R. & Cerecetto, Hugo & Chen, Peng \\
\hline Canevotti, Renato & Ceron, Jose & Chen, Pin-Shern \\
\hline Canini, Antonella & Chabert, Philippe & Chen, Qi \\
\hline Cantorna, Margherita & Chan, Chin-Feng & Chen, Ruey-Shyang \\
\hline Cao, Jay J. & Chan, Shun-Wan & Chen, S. \\
\hline Cao, Song & Chan, Stephen L. & Chen, Wei-Min \\
\hline Cao, Yanping & Chan, Thomas Y. K. & Chen, Wen-Bin \\
\hline Caporale, A. & Chang, Cicero L. T. & Chen, Xi \\
\hline Cappelli, Chiara & Chang, Fang-Rong & Chen, Xuanmao \\
\hline Caputo, Gregory A. & Chang, Hsueh-Wei & Chen, Yen-Ling \\
\hline Caraglia, Michele & Chang, Hyeun Wook & Chen, Ying \\
\hline Caramella, Carla & Chang, Il-Moo & Chen, Yue \\
\hline Cardile, V. & Chang, Raymond & Chen, Zhe-Sheng \\
\hline Cardoso, Susana M. & Chang, Sandra P. & Cheng, Dandan \\
\hline Carloni, Paolo & Chang, Sue Joan & Cheng, Hsueh-Ling \\
\hline Carmona, Daniel & Chang, Tom & Cheng, Kuan-Chen \\
\hline Carradori, Simone & Chang, Young-Tae & Cheng, Ming-Jen \\
\hline Carrano, Carl J. & Chang, Yuan Shiun & Cheng, Tak \\
\hline Carrasco-Pozo, Catalina & Chantrapromma, Suchada & Cheng, Tiejun \\
\hline Carrillo, María Cristina & Chapman, Eli & Cheng, Wei-Chieh \\
\hline Carroll, Camille B & Chaves, Mariana & Cheng, Xin-Lu \\
\hline Carta, Antonio & Chen, Baohua & Cheng, Yiyun \\
\hline Caruso, Francesco & Chen, Calvin Yu-Chian & Cheng, Yongxian \\
\hline Carvajal-Millan, Elizabeth & Chen, Chiachung & Cheong, JaeHun \\
\hline Carvalho, Ivone & Chen, Chien-Chih & Chang. Chi-I. \\
\hline Casas, L. & Chen, Chung-Yi & Chi, Robin Yonggui \\
\hline Casemiro, Luciana Assirati & Chen, D. & Chia, Brian \\
\hline Caskey, Charles F. & Chen, Fadi & Chiang, Chiao-Hsi \\
\hline Castagné, Vincent & Chen, Haifeng & Chiang, Hung-Lung \\
\hline Castellano, José María & Chen, Hubiao & Chiang, John \\
\hline Castellari, Massimo & Chen, Hui-Sheng & Chiba, Kazuhiro \\
\hline Castellarin, Simone D. & Chen, Ih-Sheng & Chibiryaev, A. M. \\
\hline Castro, Angeles & Chen, Jhy-Der & Chien-Chih, Chiu \\
\hline
\end{tabular}




\begin{tabular}{|c|c|c|}
\hline Chien, Yi-Chi & Cock, Ian E. & Cuerva, Juan M. \\
\hline Chiou, Bor-Sen & Cody, Jeremy & Culmsee, Carsten \\
\hline Chiou, Shih-Hwa & Coelho, Jaime & Cultrone, Antonietta \\
\hline Chiou, Wen-Fei & Coiffard, Laurence J. M. & Cumpstey, Ian \\
\hline Chirea, Mariana & Colca, Jerry R. & Cunha, Anna C. \\
\hline Chiti, Arturo & Coleman, Anthony W. & Cunico, Wilson \\
\hline Chmielewski, Marek & Coll, Josep & Curcio, Manuela \\
\hline Cho, Chan Sik & Collins, Shawn K. & Custodio, Luisa \\
\hline Cho, Yong Seo & Colom, Helena & Cuzaocrea, Salvatore \\
\hline Cho, Young Sik & Colombo, Maria Laura & Czech-Kowalska, Justyna \\
\hline Choi, Dong-Kug & Combes, Sébastien & D'abrosca, Brigida \\
\hline Choi, Hyung-Kyoon & Combrinck, S. & D'ambrosio, Michele \\
\hline Choi, Jae-Hong & Comes-Franchini, Mauro & D'Andrea, Luca D. \\
\hline Choi, Jong Hyun & Conesa, J. A. & Dahlberg, Clinton J. \\
\hline Choi, Kyoungju & Conrad, Marcus & Dai, Zong \\
\hline Choi, Seok Ki & Consoli, Grazia M. L. & Dall'Acqua, Stefano \\
\hline Choi, Yung Hyun & Contestabile, Antonio & Dallinger, Doris \\
\hline Choma, Adam & Coppola, Thierry & Damatta, Fabio M. \\
\hline Chong, Youhoon & Coquerel, Yoann & Daming, Du \\
\hline Chootip, Krongkarn & Corazza, Marcos L. & Daniele, Simoni \\
\hline Choshi, Tominari & Corrêa, Arlene & Daniels, Matthew H. \\
\hline Chou, Duen-Suey & Correia, Jack & Danishefsky, Samuel J. \\
\hline Choy, Ying & Cosconati, Sandro & Danova, Kalina \\
\hline Choyke, Peter L. & Cosgarea, Rodica & Dantas, Renato F. \\
\hline Christensen, Søren Brygger & Cossignani, Lina & Dante, Roberto C. \\
\hline Chrostowska, Anna & Cossio, Fernando & Dauncey, M. \\
\hline Chu, Fang-hua & Costa, Susana P. G. & Davies, Stephen G. \\
\hline Chu, Justin & Costoya, Jose A. & De Almeida Barbosa, L. Claudio \\
\hline Chua, Lee Suan & Couce, María D. & De Andrade e Silva, Suheyla \\
\hline Chuang, John Shih-ching & Couture, Olivier & De Beer, Dalene \\
\hline Chuman, Hiroshi & Couvineau, Alain & De Beer, J. \\
\hline Chun, Ock & Cox, Liam R. & De Carvalho, Carla \\
\hline Cicco, Nunzia & Creamer, Rebecca & De Feo, vincenzo \\
\hline Cielecka-Piontek, Judyta & Cresteil, Thierry & De Freitas, Rivelilson Mendes \\
\hline Cimanga, R. K. & Crews, Colin & De Groot, Herbert \\
\hline Cioli, Donato & Crich, Simonetta Geninatti & De Kimpe, Norbert \\
\hline Cirillo, Giuseppe & Cristofari, Gael & De La Cruz, V. Pérez \\
\hline Clarés-Naveros, Beatriz & Crowley, James D. & De La Fuente, M. A. \\
\hline Clark, Daniel A. & Crupi, P. & De La Hoz, Antonio \\
\hline Clarke, Anthony & Cruz, Jader S. & De Lago, Eva \\
\hline Clarke, Donald & Csuk, René & De Luca, Vincenzo \\
\hline Cocco, Melanie & Cuca, Luis E. & De Luna Martins, Daniela \\
\hline
\end{tabular}


De Mendonça, Dina I. M. D.

De Moliner, Fabio

De Oliveira Monteiro, Caio Márcio

De Oliveira, Helena C. F.

De Oliveira, Luiz Fernando C

De Paz, Jose Luis

De Quirós, A. Rodríguez-Bernaldo

De Robertis, Riccardo

De Sousa, Orlando Vieira

De Souza, Ana Olívia

De Souza, Gezimar D.

De Spiegeleer, Bart

De Villiers, André

De Vrese, Michael

De With, Gijsbertus

Debbert, Stefan L.

Debnath, Samir

Décaudin, Bertrand

Defrancq, Eric

Dehaen, Wim

Delgado, Francisco J.

DellaGreca, Marina

Delogu, Lucia Gemma

Delsart, Cristèle

Dembinski, Roman

Demopoulos, Vassilis J.

Denny, Bill

Denti, Michela A.

Desaulniers, Jean-Paul

Desbois, Nicolas

Desmaële, Didier

Desreux, Jean F.

Destefano, J. J.

Detrembleur, Christophe

Devesa, V.

Devi, M. Geetha

Devreese, Mathias

Dhawan, Deepika

Di, Rong

Diamond, Gill

Diana, Patrizia

Dias, Bernardo
Diaz-Lanza, Ana Maria

Diaz, Isabel

Díaz, Mario

Diez, David

Dijkstra, Pieter J.

Dijkwel, Paul

Dilecce, Giuseppe

Dilis, Vardis

Ding, Gang

Ding, Jian

Ding, Jinsong

Ding, $\mathrm{Ke}$

Ding, Xiaochu

Dinglasan, Rhoel R.

Dixit, Bharat C.

Djarova, Tryana

Dodziuk, Helena

Doligalska, M.

Domingo, Luis R.

Dominici, Paola

Doña-Rodríguez, José M.

Dondoni, Alessandro

Dong, Yuesheng

Donner, Christopher D.

Donno, D.

Donsì, Francesco

Donzello, Maria Pia

Dooley, S.

Dragutan, Ileana

Dragutan, Valerian

Drosatos, Konstantinos

Du, Rose

Du, Yuguo

Du, Yunfei

Duan, Jinao

Duarte, Whasley Ferreira

Düchler, Markus

Dueňas-González, A.

Duerkop, Axel

Duh, Pin-Der

Dumoulin, F.

Duncan, Robin E.
Duodu, Kwaku G.

Durán-Patrón, Rosa

Dwivedi, Chandradhar

Dykes, Gary A.

Easmon, Johnny

Ebel, Rainer

Ebinger, Katalin

Ebrahimi, Azadeh

Echevarria, Aurea

Eckert, Heiner

Eckl, Peter M.

Eichen, Yoav

Eichler, Jutta

Eifler-Lima, Eifler-lima

Eitsuka, Takahiro

Eklund, Patrik C.

Eklund, Per

El Kaïm, Laurent

El-Achkar, Tarek

El-Amri, Chahrazade

El-Readi, Mahmoud Zaki

El-Seedi, Hesham R.

Eller, Fred

Elmegeed, Gamal A.

Elofsson, Mikael

Elwakeel, Khalid Z.

Engels, Joachim W.

Enjoji, Munechika

Enríquez, Raúl G.

Erdélyi, Máté

Ermert, Johannes

Escalante, Jaime

Escolano, Carmen

Escriche, Isabel

Escuredo, Olga

Esnault, Vincent L. M.

Espinosa-Aguirre, Esús Javier

Esquivel, Rodolfo O.

Ess, Daniel H.

Estevinho, Leticia

Etzkorn, Manuel

Evans, Amanda C. 
Faber, Kurt

Fahmy, Hesham T. Y.

Faik, Ahmed

Fallarero, Adyary

Fan, Enguo

Fan, Mingtao

Fan, Timothy M.

Fan, Yunchang

Fan, Zhijin

Fanning, Kent

Farid, Chemat

Fariña, Julia I.

Farr, Susan A.

Fasinu, Pius S.

Fausto, Rui

Favi, Gianfranco

Fayet, G.

Feás, Xesús

Felluga, Fulvia

Felsenstein, Kevin M.

Feng, Meiqing

Feng, Yangbo

Feng, Yibin

Feo, Francesco

Feresin, Gabriela E.

Feringa, Ben L.

Fernand, Vivian

Fernandes, K. F.

Fernandez Megia, Eduardo

Fernández-Alba, A. R.

Fernández-Arroyo, Salvador

Fernandez-Lafuente, Roberto

Fernández-Miyakawa, M. E.

Fernandez-Recio, Juan

Fernández, José J.

Fernández, Rosario

Feron, Gilles

Ferrari, Stefania

Ferreira, Juliana

Ferreira, Marcelo J. P.

Ferreira, Susana

Fiala, André
Fickers, Patrick

Figadere, Bruno

Figueiredo, Ana Cristina

Filarowski, Aleksander

Filippov, Dmitri V.

Fiorucci, Stefano

Fischer, Reinhard

Flaherty, Patrick T.

Fliegner, Daniela

Florea, Bogdan I.

Florence, Val

Fondjo, Emmanuel Sopbué

Font, A.

Font, María

Fontana, Antonella

Fontes, Ana

Forano, Evelyne

Fornari, Francesca

Fornari, Tiziana

Fortunatia, E.

Foster, J. A.

Foti, Robert S.

Foubelo, Francisco

Fragai, Marco

Francia, Silvia De

Francis, Heather

Francis, I. Leigh

Franco, Octávio Luiz

Frappier, Lori

Frau, Juan

Freile, Mónica L.

Freitas, Ana Cristina

Fristrup, Peter

Froldi, Guglielmina

Frolov, Andrej

Frontera, Antonio

Frontier, Alison J.

Fryxell, Glen

$\mathrm{Fu}$, Hua

$\mathrm{Fu}, \mathrm{Li}-\mathrm{Wu}$

$\mathrm{Fu}$, Shaohai

$\mathrm{Fu}$, Tzu-Fun
Fu, Zhifeng

Fuguet, Elisabet

Fujii, Katsuhiko

Fujimoto, Yoshinori

Fujita, Masaki J.

Fujita, Yoshio

Fukuda, Michiko

Fukusaki, Eiichiro

Fukuyama, Tomoki

Fulgosi, Hrvoje

Furuzono, Tsutomu

Fuselli, Fabio

Fusi, Vieri

Fuwa, Haruhiko

Gabriele, Bartolo

Galagudza, Michael

Galán-Vidal, Carlos

Galat, A.

Galati, Enza Maria

Galderisi, Umberto

Gali-Muhtasib, Hala U.

Gallagher, John F.

Galvão, Adelino M.

Gambino, Dinorah

Gamcsik, Michael P.

Gamel, Tamer

Gan, Liangbing

Gandhi, Neha

Gannett, Peter

Gao, Yanxiang

Gao, Feng

Gao, Haixiang

Gao, Jin-Ming

Gao, Kun

Gao, Min

Gao, Shuanhu

Gao, Wen-Yuan

Gao, Xiumei

Gao, Yuan

Garaj, Vladimír

GarcÃ-a-Domenech, Ramon

García-Álvarez, Joaquín 


\begin{tabular}{|c|c|c|}
\hline García-Martinez, Joaquín C. & Gimpl, Gerald & Graether, Steffen P. \\
\hline García-Parrilla, M. C. & Gioiello, Antimo & Graf, Robert \\
\hline García-Valverde, María & Giralt, Ernest & Grandas, Anna \\
\hline García-Villanova, Belén & Girbés, Tomás & Granet, Robert \\
\hline Garcia, R. & Gising, Johan & Granica, Sebastian \\
\hline Garozzo, Adriana & Giuffrida, Daniele & Grant, Stephan \\
\hline Garraud, Olivier & Giunchedi, Paolo & Grassi, Alfonso \\
\hline Garre, Dulcenombre Gómez & Glaser, Keith B. & Grassi, Mario \\
\hline Garrote, Gil & Glauser, Gaetan & Gratteri, Paola \\
\hline Gáspári, Zoltán & Gledhill, Martha & Gratzl, Miklos \\
\hline Gaunitz, Frank & Gobis, Katarzyna & Graves, David \\
\hline Gauthier, Charles & Godt, Adelheid & Green, Ezekiel \\
\hline Gawlik-Dziki, Urszula & Gomes, Valdirene Moreira & Greenberg, Robert M. \\
\hline Gawrisch, Klaus & Gómez-Caravaca, Ana María & Greenberger, Joel S. \\
\hline Gbogouri, Albarin & Gómez-Gómez, Lourdes & Greer, Charles August \\
\hline Geierstanger, Bernhard $\mathrm{H}$. & Gonçalves, M. Sameiro T. & Greisch, Jean-Francois \\
\hline Gein, V. L. & Gong, Xingchu & Grenier, Nicolas \\
\hline Gelmi, Maria Luisa & Gong, Young-Dae & Grieser, Franz \\
\hline Gendaszewska-Darmach, Edyta & González-Aguilar, Gustavo Adolfo & Griffith, Renate \\
\hline Geneviève, Bourdy & González-Gómez, D. & Griffiths, William \\
\hline Gennäs, Gustav Boije Af & González-Marrero, Joaquin & Grimaldi, Maurizio \\
\hline Georgiev, Milen & González, Concepción C. & Grinberg, Sarina \\
\hline Gerbaux, Pascal & González, María Pilar & Groppo, Francisco Carlos \\
\hline Geronikaki, Athina & Goping, Ing Swie & Gros, Claude P. \\
\hline Gerós, Hernâni & Gordaliza, M. & Gruber, Christian \\
\hline Gerwick, Lena & Gordon, Michael & Grune, Tilman \\
\hline Geso, Moshi & Gorjanović, Stanislava Ž. & Gruselle, Michel \\
\hline Gestwicki, Jason E. & Gorman, Gregory S. & Grzebisz, Witold \\
\hline Ghandi, Khashayar & Gorzalczany, S. & $\mathrm{Gu}$, Yong \\
\hline Ghilardi Lago, João Henrique & Goslinski, Tomasz & Gu, Zhongwei \\
\hline Ghosh, Dipankar & Gosmann, Grace & Gude, Veera Gnaneswar \\
\hline Giancola, Concetta & Gossage, E. R. T. & Gudiminchi, Rama \\
\hline Gibbs, Roz & Goti, Andrea & Guénin, Erwann \\
\hline Giernoth, Ralf & Gotor-Fernández, Vicente & Guerrero, Sergio A. \\
\hline Gikas, E. & Gottesman, Michael M. & Guidi, Lucia \\
\hline Gil, Jose' I. & Gottfried, Carmem & Guil-Guerrero, Jose \\
\hline Gilani, Anwar & Gou, Shao-Hua & Guilhon, Giselle Maria S. P. \\
\hline Gilmer, John F. & Gourier, Didier & Guillaume, Dominique \\
\hline Gimalova, F. A. & Gourley, Shannon L. & Guillemette, T. \\
\hline Giménez, Dolores García & Goya, Luis & Guillemin, Jean-Claude \\
\hline Giménez, Julieta Pérez & Grabchev, Ivo & Gülçin, İlhami \\
\hline Giménez, María S. & Graber, David J. & Gunstone, Frank D. \\
\hline
\end{tabular}


Guo, Jia

Guo, Ju-Tao

Guo, Qiaosheng

Gurvich, Vadim J.

Gutiérrez-Uribe, Janet A.

Gutierrez, Rosa Martha Perez

Guzmán, Esther A.

Hackenberg, Michael

Hada, Kihito

Hadad, Mohamed

Hadjipavlou-Litina, Dimitra

Haertlé, Thomas

Haider, Norbert

Haiges, Ralf

Haino, Takeharu

Hak, Sjoerd

Hakamata, Hideki

Halasz, Ivan

Halford, Nigel G.

Hall, Marilena

Hallouard, François

Hamana, Hiroshi

Hamid, N.

Hamme Ii, Ashton T.

Hampel, Daniela

Han, Jaehong

Han, Jin

Han, Quan-Bin

Han, Sang-Bae

Han, Weidong

Handa, Avtar K.

Hanessian, Stephen

Hanhineva, Kati

Hano, Christophe

Hanrahan, Grady

Hansen, D. Flemming

Hansen, Michael Ryan

Hanson, Susan K.

Hao, Da-Cheng

Harding, Wayne W.

Harper, Jason B.

Harris, Philip
Harrison, William

Hartley, C. Scott

Hartmann, Martin

Hasegawa, Tomonobu

Hashimoto, Masaru

Hassan, Mohamed

Hatae, Noriyuki

Hatakeyama, Susumi

Hatano, Tsutomu

Havet, Jean-Louis

Hayashi, Yoshihiko

Haybaeck, Johannes

He, Hong-Ping

He, Langchong

He, Liangnian

He, Xiao-Peng

He, Yanhong

Hearn, Michael J.

Heasley, Brian H.

Hedges, Jodi

Hegedüs, A.

Heilig, Andrej

Henary, Maged

Henderson, Luke

Henryk, Kozlowski

Heras, Ángeles

Herczegh, Pál

Herdewyn, Piet

Herling, Marco

Hermosa, Rosa

Herrador, M. Mar

Herraiz, T.

Heuberger, Eva

Heuzey, Marie-Claude

Hider, Robert C.

Higman, Vicky

Hijji, Yousef

Hindsgaul, Ole

Hipkiss, Alan R.

Hirano, Tomoya

Hirao, Akira

Hirayama, Masao
Hiroaki, Hidekazu

Hiruma-Lima, Clelia

Ho, Chi-Tang

Ho, Feng-Ming

Ho, Mei-Lin

Ho, Su-Chen

Höbartner, Claudia

Hoffman, Sebastien

Hofmann, Alan F.

Hogg, Philip

Hohlfeld, Thomas

Hojo, Hironobu

Højrup, Peter

Hollung, K.

Holmgren, Arne

Holzgrabe, Ulrike

Honda, Hisashi

Honda, Tadashi

Hondal, Robert J.

Hong, Jiann-ruey

Hong, Jongki

Hong, Liangzhi

Hong, Mei

Hong, Yonggeun

Honker, Hans

Horng, Jim-Tong

Horobin, Richard W.

Hort, Mariana Appel

Hoshino, Tyuji

Hosni, Karim

Hossain, Mohammad B.

Hou, Chien-Wen

Hou, Wen-Chi

Howard, Zack

Howell, Amy R.

Howes, Melanie

Hozák, Pavel

Hrckova, Gabriela

Hromadkova, Z.

Hseu, You-Cheng

Hsieh, Hsing-Pang

Hsieh, Jen-Chien 
Hsieh, Yi-Hsien

Hsing, C.-H.

Hsu, Day-Shin

$\mathrm{Hu}$, Bing

$\mathrm{Hu}$, Miao-Lin

$\mathrm{Hu}$, Miao-Lin (Merlin)

$\mathrm{Hu}$, Tianhui

$\mathrm{Hu}$, Wei-Wen

$\mathrm{Hu}$, Xiang-Ping

$\mathrm{Hu}$, Xiaowen

$\mathrm{Hu}$, Yonghong

$\mathrm{Hu}, \mathrm{Zhe}-\mathrm{Yi}$

Hua, Daoben

Hua, Erbing

Hua, Huiming

Huang, Chang-Gan

Huang, Chi-Chang

Huang, Feihe

Huang, Jinhua

Huang, Mingdong

Huang, Xuefei

Huang, Ya-Ling

Huang, You

Huang, Zhen

Huang, Zhibin

Huber, Thomas

Huczyński, Adam

Hudson, Bruce

Hui, Kam M.

Hulme, Christopher

Hunger, Johannes

Hunyadi, Attila

Huq, Fazlul

Hurvois, Jean-Pierre

Hussain, A.

Hwang, Baik

Hwang, Kyung A.

Hwu, Jih Ru

Hyafil, Fabien

Hyun, Myung Ho

Ibrahim, S. A.

Ichihashi, Masamitsu
Ifa, Demian

Iglésias, Bernardo

Ikejima, Kenichi

Im, Sin-Hyeog

Imai, Shinjiro

Imbimbo, Bruno P.

Immenschuh, Stephan

Inamoto, Kiyofumi

Inoue, Koichi

Iorizzi, Maria

Iqbal, Zafar

Isaksson, Dan

Isasi Allica, José Ramón

Ishibashi, Masami

Ishihara, Kazuaki

Ishikawa, Tomoyasu

Ishikura, Minoru

Ishimoto, Takayoshi

Ishman, P. F

Islam, Shahidul

Ismaiel, Omnia

Isman, M. B.

Isshiki, Yoshiaki

Ito, Hiroyasu

Ito, Takeo

Itoh, Ken-Ichi

Ittner, Lars $\mathrm{M}$.

Ivanov, Ivo C.

Ivanovska, Nina

Iwabuchi, Yoshiharu

Iwagawa, Tetsuo

Iwamori, Masao

Iwashina, Tsukasa

Izquierdo, Luis

Jaakola, Laura

Jacqz-Aigrain, Evelyne

Jafarpour, Farnaz

Jagerovic, Nadine

Jagodziński, Paweł P.

Jampilek, Josef

Jänis, Janne

Jarosz, Slawomir
Jäschke, Andres

Jaworska, Malgorzata

Jean De Dieu, Tamokou

jean, Ludovic

Jeandet, Philippe

Jeng, Ru-Jong

Jensen, Søren Rosendal

Jeon, Jae-Gyu

Jeon, You-Jin

Jeong, Hyun-Ja

Jessen, Henning Jacob

Jezek, Jan

Ji, Shun-Jun

Jia, Cheng-Sheng

Jia, Xinru

Jiang, Hongliang

Jiang, Lin

Jiang, Xuchuan

Jiang, Yuyang

Jianhui, Qiu

Jiao, Guangling

Jim Simpson, Jim Simpson

Jimbo, Mitsuru

Jiménez-Barbero, Jesús

Jiménez, Jara Pérez

Jimeno, Ciril

Jin, Lijian

Jin, Weijun

Joe, Young Ae

Johansen, Kenneth T.

Johnson, Jeremy J.

Johnson, John A.

Jones, Christopher E.

Jonnalagadda, Subash C.

Joo, Myungsoo

Jordan, Joaquin

Jordão, António M.

Jóźwiak, Krzysztof

Juillerat-Jeanneret, Lucienne

Jullian, Valérie

Jung, Jiyoung

Jung, Youngmi 
Junkers, Thomas

Juszczak, Lesław

Kachlicki, Piotr

Kai, Guoyin

Kajihara, Yasuhiro

Kakkar, Ashok K.

Kaliora, Andriana C.

Kalogeropoulos, Nick

Kalvins, Ivars

Kamel, Alaa

Kami, Daisuke

Kamio, Michiya

Kammerer, Robert

Kamolz, Lars P.

Kanakis, George

Kang, ChulHee

Kang, Congbao

Kang, Dawon

Kang, Jonghoon

Kang, Sam Sik

Kapoor, Mahendra P.

Karagiannis, Tom C.

Karvembu, R.

Kashiwada, Yoshiki

Kästner, Johannes

Kataoka, Takao

Kataoka, Yosky

Katarg, Argyropoulou

Katayama, Hidekazu

Kato, Eisuke

Kato, Masako

Katuchova, Jana

Kaurinovic, Biljana

Kawabata, Saneyuki

Kawabe, Takefumi

Kawanishi, Noriaki

Kay, Brian K.

Kazakova, O. B.

Keller, Thomas H.

Kendrick, John

Kerkhoff, Claus

Kerr, Phil
Kerwin, Sean M.

Keul, Helmut

Keyzers, Rob

Khalaf, Abedawn I.

Khalifa, Mohamed E.

Khan, Khalid Mohammed

Khan, N. A.

Kharwar, Ravindra

Khasawneh, Mohammad

Kikuzaki, Hiroe

Kim, Bumseok

Kim, Dong-Myung

Kim, Guntae

Kim, Hyung Min

Kim, Jin

Kim, Jin Moon

Kim, Jin Sook

Kim, Jung-Ae

Kim, Junheon

Kim, Minsoo

Kim, Nacksung

Kim, Soo-Un

Kim, Sung Yeon

Kim, Sung-Hoon

Kim, Uh-Hyun

Kim, Y. D.

Kim, Yeong Shik

Kim, Youngmee

Kimata-Ariga, Yoko

Kimura, Hideto

Kingsbury, Joanne M.

Kirby, Anthony

Kirby, Stephen D.

Kirsch, Gilbert

Kiso, Makoto

Kiso, Yoshiaki

Kiss, Anna K.

Kitamura, Tsugio

Kiuru, Paula

Kjaer, Anders

Klajnert, B.

Klapper, M.
Klein, Christian D.

Kleinpeter, Erich

Klinman, Judith P.

Klochkov, S. G.

Kloucek, Pavel

Kluza, J.

Knapp, Spencer

Knauer, Shirley K.

Knölker, Hans-Joachim

Knoshaug, Eric

Knudsen, Lotte Bjerre

Kobayashi Japan, Hidekazu

Kobayashi, Keiji

Kobayashi, Yuichi

Kodama, Hiroyuki

Kohaar, Indu

Kohen, Amnon

Kojima, Akiko

Kojima, Chie

Kojima, Chojiro

Kojo, Shosuke

Kolaczkowski, Marcin

Kolbe, L.

Kolehmainen, Erkki

Kolodziejczyk, Joanna

Komar, Anton A.

Kong, De-Ming

Kong, Dejuan

Kong, Ling-Yi

Konrath, Eduardo Luis

Koóš, Miroslav

Kopka, Klaus

Kopp, B.

Koppel, Kadri

Košak, Aljoša

Koschella, Andreas

Kosterink, Jos G. W.

Kostikov, Alexey P.

Kostogrys, Renata

Kotani, Shunsuke

Koyama, Tomoyuki

Kozikowski, Alan P. 
Kozliak, Evguenii I.

Kozlov, Igor A.

Kozlowski, Henryk

Krajcsi, Peter

Krämer, Oliver H.

Kredics, Laszlo

Kremer, Dario

Krstic, Natalija

Kruger, Marlena

Kuang, Shihuan

Kubíček, Vojtěch

Kubo, Takuya

Kubo, Yuji

Kubota, Yasuhiro

Kucharikova, Sona

Küçükbay, Hasan

Kühn, Fritz E.

Kühn, Oliver

Kukovec, Boris-Marko

Kulik, Eva M.

Kulisic-Bilusic, Tea

Kulozik, Ulrich

Kumar, Manoj

Kumar, Rakesh

Kumar, Sanjai

Kumar, Satish

Kung, Hank F.

Kuniyasu, Akihiko

Kuo, Ping-Chung

Kuo, Sheng-Chu

Kuo, Yao-Haur

Kupcewicz, Bogumiła

Kurek, Mia

Kuroda, Minpei

Kurtán, Tibor

Kurtboke, Ipek

Kushida, Hirotaka

Kushiro, Tetsuo

Kvansakul, Marc

Kwon, Il-Keun

Kwon, Jung-Hwan

Kwon, Young-In
Kwon, Youngjoo

Kwong, Fuk Yee

La Clair, James

La Cruz, Thomas E.

Lacaille-Dubois, Marie-Aleth

Lafont, René

Lai, Chih-Ho

Lai, Daowan

Lai, Hung-Cheng

Lai, Jui-Yang

Lam, Jacky W. Y.

Lämmerhofer, Michael

Lammich, S.

Lang, Siegmund

Lange, Oliver

Langer, Oliver

Langer, Swen

Laplante, Mathieu

LaPlante, Steven R.

Larionov, Oleg V.

Larsen, Scott D.

Lasunción, Miguel A.

Laufer, Stefan

Lauria, Antonino

Lavine, Barry

LaVoie, Edmond J.

Lawson, Charlotte

Lazar, Diana

Lazar, S.

Lazareva, Nataliya F.

Lazaridis, Themis

Lazzarato, Loretta

Leardkamolkarn, Vijittra

Lebovka, N. I.

Lee, Dong Gun

Lee, Francis Young-In

Lee, Hangu

Lee, Hsinyu

Lee, Hyi-Seung

Lee, Jae Yeaol

Lee, Jae-Ho

Lee, Jaehwi
Lee, Jeong-Chae

Lee, Jin-Ching

Lee, Jin-Woo

Lee, Jiseok

Lee, Kyung-Yeol

Lee, Mi Kyeong

Lee, Min

Lee, Moses

Lee, Myung G.

Lee, Ok-Hwan

Lee, Rebecca

Lee, Ren-Shen

Lee, Seokjoon

Lee, Shim Sung

Lee, Soo Young

Lee, Zang Hee

Lee, Zhi-Hong

Legraverend, Michel

Lehmler, Hans-Joachim

Lehocky, Marian

Lei, Pingsheng

Lei, W.

Lelonek, Malgorzata

Len, Christophe

Lenardão, Eder J.

Lentini, Giovanni

Lentjes, E. G. W. M.

León-Rivera, Ismael

Leonard, C. M.

Leonelli, Francesca

Leonetti, Francesco

Leong, Lai Peng

Leonidas, Demetres D.

Lépine, Francis

Lertsiri, Sittiwat

Lespade, Laure

Lesyk, Roman

Leu, Yann-Lii

Leung, Chung-Hang

Leung, Dennis H.

Leung, Euphemia

Leung, Kar W. 
Leung, Pc

Lewinska, Anna

Lewis, Gareth

Li, Cheng

Li, Chien-Ming

Li, Chuan

Li, Chuangchuang

Li, Dehai

Li, Fuyou

Li, George Qian

Li, Huabin

Li, Huaming

Li, Huihui

Li, Jian

Li, Jianrong

Li, Jianshu

Li, Jing

Li, Kecheng

Li, Lin

Li, M. X.

Li, Meng-Hui

Li, Nianguang

Li, Ning

Li, Peiwu

Li, Pengcheng

Li, Rui

Li, Shao-Shun

Li, Shenshen

Li, Wei

Li, Xiaoyu

Li, Yanfeng

Li, Yaping

Li, Yi

Li, Yiming

Li, Yingwei

Li, Yuliang

Li, Zibo

Liang, Po-Huang

Liang, Tingbo

Liang, Xin-Miao

Lião, Luciano M.

Liao, Wuping
Liao, Yang

Liao, Yong-Hong

Liebscher, Juergen

Liekens, Sandra

Liese, Andreas

Liguori, Angelo

Lillich, James D.

Lim, Beong Ou

Lim, Yau-Yan

Lim, Yoongho

Lima, Lídia Moreira

Lin, Chih-Chien

Lin, Chun-Cheng

Lin, Chun-Ching

Lin, Chun-Yuan

Lin, Hai-Shu

Lin, Hsiang-Ru

Lin, Jingming

Lin, Kuo-I.

Lin, Nai-Chun

Lin, Shyh-Hsiang

Lin, Tzu-Chau

Lin, Wen-Han

Lin, Xufeng

Lin, Ying-Chih

Lin, Yun-Lian

Lin, Yun-Wei

Lin, Zhenjian

Linclau, Bruno

Lincoln, Per

Lindhorst, Thisbe

Lindoy, Leonard

Ling, Jian Ya

Ling, Li

Liou, Jing-Ping

Liou, Kuo-Tong

Liskamp, Rob M. J.

Listrat, Anne

Litinas, Konstantinos

Litonjua, Augusto A.

Litvić, Mladen

Litwinienk, Grzegorz
Liu, Fang

Liu, Hai-Yang

Liu, Ji-Bin

Liu, Jiang-Xun

Liu, Jie

Liu, Jingze

Liu, Lei

Liu, Liang

Liu, Pengzhan

Liu, Ruifeng

Liu, Sean X.

Liu, Shing-Hwa

Liu, Yonghong

Liu, Yongjian

Liu, Yongjun

Liu, Yung-Chuan

Liu, Yunling

Liu, Zai-Qun

Liu, Zhi Long

Liu, Zhi-Pei

Liu, Zhongfa

Lizard, Gérard

Lo Scalzo, Roberto

Lo, Jeng-Fan

Lobo-Castañón, Maria Jesus

Locatelli, Marcello

Lodovici, Maura

Longley, Db

Loo, Alvin

Lopes, Dinora

López, Carlos Silva

López, Carmen Remuñán

Lopez, Fernando

López, Luis A.

López, Víctor

Lorenz, Peter

Los, Marek

Löser, Reik

Lotina-Hennsen, Blas

Lovero, Grazia

Lowary, Todd L.

Lowe, Gordon 
Lu, Chengping

Lu, Chung-Dar

Lu, Dah-Yuu

Lu, Jin-Jian

$\mathrm{Lu}$, Jun

Lu, Long

Lu, Mangeng

Lu, Pei-Luen

$\mathrm{Lu}$, Rong

Lu, Wei

$\mathrm{Lu}$, Yong

$\mathrm{Lu}$, Yunhua

Lu, Zhaoxin

Lucas, Pierre

Lucinda, Leda Marília Fonseca

Lüdtke, Diogo S.

Ludvík, Jiří

Lugo-Cervantes, Eugenia

Lui, Ho Chi

Luo, Qun-Li

Lupiáñez, José A.

Luque, Rafael

M. Nyström, Andreas

Ma, Chaomei

Ma, Cheng

Ma, Choong

Ma, Edmond Dik-Lung

Ma, Enlong

Ma, Guang-Hui

Ma, Sen

Ma, Shengqian

Macleod, Roderick A. F.

Maddocks, Sarah E.

Madej, Katarzyna

Maeda, Kiminori

Maeda, Toshinari

Maes, Louis

Maffei, Massimo

Maga, Giovanni

Magazu, Salvatore

Magedov, Igor V.

MaGee, David I.
Maggi, F.

Magid, Abdulmagid Alabdul

Mahana, Carlos David Pessoa

Mahatthananchai, Jessada

Maher III, L. James

Maher, Pamela

Maia, Cristiane Do Socorro Ferraz

Mak, Nai K.

Makala, Levi H. C.

Mäki-Arvela, Päivi

Mäkinen, Ville-Petteri

Makker, Pratima Nangia

Makriyannis, Alexandros

Maksimov, I. V.

Malchiodi, Emilio L.

Malekinejad, $\mathrm{H}$.

Malheiro, Ricardo

Malo, C.

Malterud, Karl Egil

Mamat, Constantin

Mambu, Angèle Lengo

Mangalagiu, Ionel I.

Mani, Sridhar

Manna, Prasenjit

Manoury, Eric

Mantegazza, Francesco

Mantell, C.

Manzhos, Sergei

Mao, Jian-Hua

Mao, Jingdong

Mara, Freire

Maranzana, Andrea

Marchal, Juan A.

Marchan, Jonathan S.

Marchioni, Eric

Marco-Contelles, José

Margarity, Marigoula

Mariano-Oliveira, Andréa

Marik, Jan

Marin, M. Luisa

Marini-Bettolo, Rinaldo

Markopoulos, John
Markopoulou, Olga

Markwick, Phineus R. L.

Marnett, Larry J.

Marra, Alberto

Marrubini, Giorgio

Marsh, Ken

Marsolais, Frédéric

Marti, Sergio

Martín-Cordero, Carmen

Martín-Mazuelos, Estrella

Martin, Daniel

Martin, Rachel

Martin, Santiago

Martinelli, Adriano

Martínez-Velázquez, Moisés

Martinez, Ana

Martínez, Ana

Martínez, Ana M.

Martínez, Tomas

Martino, Sabata

Martins, Albino

Martins, Carlos

Maruyama, Toru

Marzaro, Giovanni

Masanori, Terasaki

Massiot, Georges

Masu, Hyuma

Mata, I.

Mata, Jose A.

Matan, Narumol

Materska, Małgorzata

Mathesius, Ulrike

Mathew, Thomas

Matich, Adam J.

Matlawska, Irena

Matos, Maria J.

Matosiuk, Dariusz

Matsuda, Hisashi

Matsuda, Yudai

Matsuo, Yosuke

Matsuura, Hideyuki

Matus, José Tomás 
May, Brian

Mayr, Christine

Mazerska, Zofia

Mazutti, Marcio A.

Mazzeo, Mina

Mbeunkui, Flaubert

McAlpine, Shelli

McCarthy, J. M.

Mcdougall, Gordon J.

Mckallip, Robert J.

McKee, Tawnya C.

Mckenzie, Iain

Mclean, John H.

Medana, Claudio

Mehmetoğlu, Ülkü

Mehrvar, Mehrab

Meierjohann, Axel

Meijer, Bert

Meiler, Jens

Melagraki, Georgia

Mellor, Andrew L.

Menaa, Farid

Mencherini, Teresa

Menezes, José

Meng, Fan-Yi

Merino, Pedro

Mesías, Marta

Messeguer, Angel

Messori, Luigi

Meyer, Matthew P.

Meyer, R.

Miao, Lu

Miao, Zhiwei

Miceli, Antonio

Michaelakis, Antonios

Michele, Baglioni

Micura, Ronald

Miguel, M. G.

Mikkelsen, Deirdre

Mikulski, Damian

Milewski, Sławomir

Milhauser, Glenn
Miller, Stephen C.

Mimaki, Yoshihiro

Min, Byung Sun

Minoura, Mao

Minto, Robert E.

Miomandre, Fabien

Miquel, Pons

Miranda, L.

Mireles Dewitt, Christina A.

Misra, Devesh

Misra, Sougat

Mitsunaga, Tohru

Miura, Yutaka

Miyata, Okiko

Miyazaki, Kouji

Miyoshi, Daisuke

Mizuno, Masashi

Mladěnka, Přemysl

Młochowski, J.

Mlostoń, Grzegorz

Moberg, Christina

Moderhack, Dietrich

Modica, Maria

Moeslinger, Thomas

Mohallem, Nelcy Della Santina

Mohammad, Ramzi M.

Mohan, Ram S.

Mohareb, Raafat

Moine, Laurence

Moineau-Chane-Ching, Kathleen I.

Moliner, Vicent

Möller, Heiko

Molnár, Péter

Monchaud, David

Moncol, Jan

Monge, David

Monopoli, Antonio

Montange, Damien

Montaut, Sabine

Monte, Francisco

Monteiro, Luís S.

Montesarchio, Daniela
Montón, Helena

Montoro, Paola

Moodley, Brenda

Moon, Hyung Ryong

Moon, Young-Choon

Moorthy, N. S. Hari Narayana

Moosmann, B.

Morales, Melissa

Morán, Lara

Morel, Ademir F.

Morel, Nicole

Moreno, Silvia

Moretto, Alessandro

Morgado, Jorge

Mori, Seiji

Morikawa, Toshio

Morris, John Steven

Mort, John S.

Moshi, M. J.

Moss, Mark

Motoo, Yoshiharu

Motti, Cherie

Motz, Vicki Abrams

Mourtas, Spyros

Moussaoui, Y.

Moutsatsou, P.

Moyano, Albert

$\mathrm{Mu}$, Linjing

Muddiman, David C.

Mueller-Harvey, Irene

Muhle, Claudia

Muise-Helmericks, Robin C.

Mulder, Patrick P. J.

Muller, C. J. F.

Muller, Christa

Müller, Thomas J. J.

Müller, Werner E. G

Murakami, Hiroto

Murakami, Manabu

Murali, D.

Murata, Toshihiro

Murkin, Andrew S. 


\begin{tabular}{|c|c|c|}
\hline Murphy, Cormac D. & Nezamzadeh-Ejhieh, Alireza & Ohnishi, Masatoshi \\
\hline Murru, Siva & Ngouela, Silvère & Ohno, Sho \\
\hline Musabayane, C. T. & Ni, Bukuo & Ohshima, Hiroshi \\
\hline Musci, M. & Nicasio-Torres, María Del Pilar & Ojeda, Manuel \\
\hline Musiol, Robert & Nicaud, Jean-Marc & Ojika, Makoto \\
\hline Mustafa, Mohd Rais & Nicoletti, Ferdinando & Oka, Natsuhisa \\
\hline Muthusamy, Visalini & Niedziałkowski, Paweł & Okada, Yoshinori \\
\hline Muzart, Jacques & Nihei, Ken-Ichi & Okada, Youji \\
\hline Nagai, Yoshinori & Nikolova, Milena & Okazaki, Toshiro \\
\hline Nagasawa, Kazuo & Nindo, Caleb & Okun, Ilya M. \\
\hline Naha, Pratap C. & Nishihara, Yasushi & Okuyama, Ryuhei \\
\hline Naidoo, Yougasphree & Nishiwaki, Hisashi & Olafsdottir, Elin Soffia \\
\hline Naila, Aishath & Nishiwaki, Nagatoshi & Olajide, Olumayokun A. \\
\hline Naito, Yuji & Nishiyama, Yasuhiro & Olar, Rodica \\
\hline Najahi, Ennaji & Nishizono, Naozumi & Oliveira, Beatriz P. P. \\
\hline Najdanovic-Visak, Vesna & Niu, Zhenbin & Oliveira, Rita De Cássia M. \\
\hline Nakaki, Toshio & Nkenke, Emeka & Oliveira, Ronaldo Lopes \\
\hline Nakamura, Kazuhiko & Nobili, Stefania & Olivier, Denise K. \\
\hline Nakamura, Mkikio & Nordén, Bengt & Olmo, Esther Del \\
\hline Nakamura, Yoshimasa & Norimoto, Hisayoshi & Oltra, J. Enrique \\
\hline Nakazawa, Jun & Nostro, Pierandrea Lo & Omata, Kenji \\
\hline Namba, Kosuke & Novellino, Ettore & Omori, Alvaro Takeo \\
\hline Nanjundaswamy, Ananda & Novo, Salvatore & Opletalova, Veronika \\
\hline Naota, Takeshi & Novotarskyi, Sergii & Orbán, Norbert \\
\hline Nardoni, S. & Ntalli, Nikoletta G. & Ortega, Angel L. \\
\hline Nau, Werner & Numata, Keiji & Ortona, Elena \\
\hline Navacchia, Maria Luisa & Nwaehujor, Chinaka & Orzechowski, Arkadiusz \\
\hline Navarrete-Vázquez, Gabriel & Nwe, Nitar & Osakada, Kohtaro \\
\hline Naviglio, Silvio & Nyokong, Tebello & Osanai, T. \\
\hline Nawwar, M. A. & O'connor, Kevin E. & Osheroff, Neil \\
\hline Nayak, Sukanta & O’Keefe, S. F. & Ośmiałowski, Borys \\
\hline Nazzaro, Filomena & Oancea, Elena & Osumi, Kenji \\
\hline Ndip, R. N. & Obenland, David & Otsuka, Hideaki \\
\hline Nechifor, Marioara & Obora, Yasushi & Overduin, Michael \\
\hline Neergheen, Vidushi & Obushak, N. D. & Pace, Andrea \\
\hline Negri, Giuseppina & Odell, Luke R. & Pacifico, Severina \\
\hline Nehira, Tatsuo & Ogawa, Akiya & Pacios, Luis \\
\hline Nerín, C. & Ogihara, Jun & Paczesny, Sophie \\
\hline Nesterov-Mueller, Alexander & Oh, Dong-chan & Padmaperuma, Asanga B. \\
\hline \multicolumn{2}{|c|}{ Nevárez-Moorillón, Guadalupe Virgi@ił, Jiyoung } & Pagan, Rafael \\
\hline Neves, Graça & Oh, Sei-Ryang & Pagano, Bruno \\
\hline Das Neves, J. & Oh, Seikwan & Page, Tony \\
\hline
\end{tabular}




\begin{tabular}{|c|c|c|}
\hline Pagenkopf, Brian L. & Pawar, Rahul S. & Piaz, Fabrizio Dal \\
\hline Paller, Channing & Pawlaczyk, Izabela & Picariello, Gianluca \\
\hline Palmans, Anja R. A. & Pecul, Magdalena & Pielichowska, Kinga \\
\hline Palmeri, Mark L. & Pedraza-Chaverri, José & Pieroni, Marco \\
\hline Palomo, Claudio & Pedrosa, Rozangela Curi & Pietro, Rosemeire C. L. R. \\
\hline Palop, Juan Antonio & Pei, Dehua & Pietrucci, Fabio \\
\hline Pan, Cheol-Ho & Pei, Jian & Piga, Rosaria \\
\hline Pan, Min-Hsiun & Pelaez, José Walter & Pigge, F. Christopher \\
\hline Pan, Tai-Long & Pelegrín, Pablo & Pike, Victor W. \\
\hline Pan, Y. J. & Pelkonen, Olavi & Pinho, Olivia \\
\hline Panayiotidis, Mihalis I. & Pellegrino, Daniela & Pinna, Gérard A. \\
\hline Pandanaboina, Sahitya Chetan & Pellizzon, Cláudia Helena & Pinto, Eugénia \\
\hline Pandey, Kailash C. & Peltzer, M. & Piotrowska, Dorota G. \\
\hline Panke, Sven & Pena, Miren & Piovezan, Anna P. \\
\hline Panpranot, Joongjai & Peng, Wen-Huang & Piwowarski, Jakub P. \\
\hline Pansare, Sunil V. & Peng, Xiaohua & Planas, Joana M. \\
\hline Pant, Archana & Penissi, Alicia B. & Planas, Marta \\
\hline Panzella, Lucia & Penso, Michele & Plasencia, Javier \\
\hline Papetti, Adele & Perego, Carla & Platas-Iglesias, Carlos \\
\hline Parameswaran, Narayanan & Pereira-Netto, Adaucto Bellarmino & Plavec, Janez \\
\hline Paraskevopoulou, Adamantini & Pereira, Leonel & Plażuk, Damian \\
\hline Pariani, Giorgio & Pereira, Olívia R. & Plech, Tomasz \\
\hline Park, Il-Kwon & Pereira, Romaiana & Pletcher, Mathew T. \\
\hline Park, Ji-Yeon & Pérez-Jiménez, Jara & Plourde, Guy L. \\
\hline Park, S. H. & Perez-Jorge, Conchita & Pluskota-Karwatka, Donata \\
\hline Park, So-Young & Pérez-Lamela, Concepción & Poblaciones, Maria J. \\
\hline Park, Youngja & Pérez-Palacios, T. & Poce, Giovanna \\
\hline Parker, Kathlyn A. & Perez-Pinzon, M. A. & Podolak, Irma \\
\hline Parker, William B. & Pérez-Trujillo, Míriam & Pohanka, Miroslav \\
\hline Parkinson, Gary N. & Pérez, Andy J. & Poinsot, Verena \\
\hline Parodi, Brunella & Perez, J. J. & Polepally, Prabhakar Reddy \\
\hline Parvu, Marcel & Pericàs, Miquel A. & Poleshchuk, Oleg \\
\hline Pasantes-Morales, H. & Perrier, Aurélie & Pontoni, Ludovico \\
\hline Paschke, Reinhard & Perrin, Charles L & Popiołek, Łukasz \\
\hline Passamonti, Sabina & Perrone, Daniela & Poppe, László \\
\hline Passarella, Daniele & Perry, Nigel Brian & Poriel, Cyril \\
\hline Pataj, Zoltan & Perticaroli, Stefania & Porte, Cinta \\
\hline Patrice, Vanelle & Peruzzini, Maurizio & Porto, Ana Lúcia Figueiredo \\
\hline Paudyal, Bishnuhari & Peters, Verena & Potter, P. M. \\
\hline Paulsen, Berit Smestad & Pethe, Stéphanie & Poucheret, Patrick \\
\hline Pavan, Giovanni M. & Petitjean, Anne & Pour, Milan \\
\hline Pavelić, Sandra Kraljević & Pianet, Isabelle & Pourquier, P. \\
\hline
\end{tabular}


Powers, Joseph R.

Pozo-Bayón, Maria Angeles

Prado, Soizic

Praly, Jean-Pierre

Preuss, Kathryn E.

Price, Andrew J.

Price, Neil P. J.

Prieto, Alicia

Prins, Leonard

Proestos, Charalampos

Proschak, Ewgenij

Prosser, R. Scott

Prüß, Birgit M.

Przybylski, Cédric

Psomas, George

$\mathrm{Pu}$, Jian-Xin

$\mathrm{Pu}, \mathrm{Min}$

Puerta, M. Carmen

Puglia, Carmelo

Pulici, Maurizio

Pyne, Stephen

Qandil, Amjad M.

Qi, Chenze

Qi, Hong

Qian, Mingxing

Qian, Wenyuan

Qiao, Jennifer

Qin, Bo

Qin, Chuanguang

Qin, Xuemei

Qiu, Bo

Qiu, Ming-hua

Qiu, Shengxiang

Queiroga, Felisbina Luisa

Queiroz, Maria-João R. P.

Quock, Raymond M.

Qvit, Nir

Raczyńska, E. D.

Rádl, Stanislav

Rafii, Michael S.

Ragno, Gaetano

Rainha, Nuno
Raja, Boobalan

Ramos-Organillo, Ángel

Ramos, Márcio Viana

Rampoldi, Luca

Rand, Thomas

Randazzo, Antonio

Rani, Sudheer D.

Rao, P. N. Praveen

Raposo, M. Manuela M.

Rasekh, Manoochehr

Raspotnig, Günther

Rathore, Rajendra

Ravu, Ranga Rao

Rawal, Viresh H.

Rawel, H. M.

Rawlings, Neil D.

Rawson, Ashish

Rawson, Jeremy

Ray, Ratna B.

Read De Alaniz, Javier

Read, Daniel S.

Reany, Ofer

Regel-Rosocka, Magdalena

Regnault-Roger, Catherine

Rehse, Steven J.

Reiser, Georg

Reiser, Oliver

Rejman, Dominik

Ren, Fazheng

Ren, Hongjun

Ren, Jian-guo

Ren, Ping

Reyes, Rosa Estrada

Reyniers, Marie-franoise
Riedmaier, Irmgard

Rigano, Daniela

Rigo, Benoît

Rinaldi, A. C.

Ringkjøbing-Jensen, Malene

Rinner, Uwe

Rio, Alberto Del

Ríos, Cristóbal De Los

Rissanen, Matti P.

Rivera, Augusto

Riveros Galan, David Santiago

Rizzotto, Marcela

Robert, E.

Robert, François

Roberts, Bryan

Robles-Zepeda, Ramón Enrique

Robles, Alfonso

Robles, Eva Sánchez

Robson, Mark

Rockenbach, Ismael Ivan

Rodembusch, Fabiano S.

Rodiño, Paula

Rodrigues, Ana Lúcia S.

Rodríguez-Arcos, R.

Rodriguez-Lopez, Jose Neptuno

Rodriguez, Jaime

Rodriguez, Saleta Vazquez

Rohn, Sascha

Rojo, Javier

Rosa, Antonella

Rosa, Luiz H.

Rose, Devin

Roseiro, Luísa B.

Rosemeyer, Helmut

Ribeiro Cerqueira, Miguel Ângelo PaRerstengren, K. Johan

Ribeiro, Marcelo L.

Rotondo, Archimede

Ricardo Da Silva, Jorge

Rouanet, Jean-Max

Ricart, Susagna

Roumy, Vincent

Ricci, Alfredo

Rourke, Jonathan P.

Rice, Joseph E.

Richardson, Sarah

Richomme, Pascal
Roy, René

Roy, Sharani

Rozentsveig, Igor B. 


\begin{tabular}{|c|c|c|}
\hline Rozi, Mohamed & Samoc, Marek & Schneider, Gyula \\
\hline Rubert, J. & Sánchez Chávez, Esteban & Schnermann, Martin \\
\hline Rubis, Blazej & Sánchez-Delgado, Roberto A. & Scholz, Martin \\
\hline Rudolph, U. & Sánchez-Moreno, Manuel & Schomburg, Lutz \\
\hline Ruffoni, Barbara & Sánchez-Vioque, R. & Schreiber, Angélica Zaninelli \\
\hline Ruiz-Azuara, Lena & Sanchez, L. & Schrekker, Henri S. \\
\hline Ruiz-Gómez, Miguel J. & Sandel, Johan & Schüller, Christoph \\
\hline Rupasinghe, H. P. Vasantha & Sandoval, Claudia & Schulman, Ira \\
\hline Rupenthal, Ilva D. & Sandow, Shaun L. & Schulte, Gunnar \\
\hline Rurali, Riccardo & Sani, Marc-Antoine & Schultz, Carsten \\
\hline Russell, Mark & Sanjay, Nigam & Schulz, Margot \\
\hline Russo, Gian Luigi & Sanna, Gavino & Schumacher, U. \\
\hline Russo, Nino & Santagati, Andrea & Schuster, David I. \\
\hline Ruzicka, Ales & Santiago, Helton & Schwalbe, Harald \\
\hline Ruzza, Paolo & Santos-Silva, Alice & Schwarz, Wolfgang \\
\hline Rybakova, Yu S. & Saraiva, Lúcia M. F. S. & Schweizer, Frank \\
\hline Ryckman, Kelli K. & Saravanan, M. & Sciarini, Lorena \\
\hline Ryu, Jae-Sang & Sarbu, Coster & Scilimati, Antonio \\
\hline Saavedra, Juan M. & Sardella, Roccaldo & Scipione, Luigi \\
\hline Sackus, Algirdas & Sarkar, Biprajit & Scognamiglio, Monica \\
\hline Saczewski, Franciszek & Sarkar, Ujjal & Scorziello, Antonella \\
\hline Saeki, Tohru & Sarver, Jeff & Scott, Colin \\
\hline Sagebiel, John C. & Sassaki, Guilherme L. & Scott, Lincoln \\
\hline Sahin, Selin & Sathe, Shridhar & Scotti, Luciana \\
\hline Saiano, Filippo & Sato, Takaaki & Scovassi, Anna Ivana \\
\hline Sainlos, Matthieu & Saturnino, Carmela & Sebestik, Jaroslav \\
\hline Saito, Masaichi & Sautreau, Asmita & Secci, Daniela \\
\hline Saito, Shinichi & Savaskan, Nic E. & Seifert, Karlheinz \\
\hline Saito, Yoshihiro & Savoia, Diego & Seifert, Roland \\
\hline Saitoh, Masao & Sawaya, Alexandra & Seijas Vázquez, Julio \\
\hline Sajeva, Maurizio & Schanze, Kirk & Seitz, Oliver \\
\hline Sakagami, H. & Schatz, Jurgen & Selbo, PÅl K. \\
\hline Sakata-Haga, Hiromi & Schenning, Albertus P. H. J. & Selig, Philipp \\
\hline Salas, Cristián & Schild, Lorenz & Semple, Susan \\
\hline Salas, Juan M. & Schiller, Stefan M. & Senel, Mehmet \\
\hline Salazar-Olivo, Luis A. & Schleicher, Erwin D. & Sener-Aki, Esin \\
\hline Sale, Stewart & Schlettwein, Derck & Seo, Shigemi \\
\hline Saleem, Mohammad & Schmalz, Hans-Günther & Séraphin, D. \\
\hline Salem, Abdelfattah Z. M. & Schmeda Hirschmann, Guillermo & Serpersu, Engin H. \\
\hline Salgueiro, Li'gia & Schmidt, Jürgen & Serra, Stefano \\
\hline Salles, Christian & Schmidt, Thomas J. & Serralheiro, Maria Luísa M. \\
\hline Salvador, Marcos & Schneider, Bernd & Serrano, Carmo \\
\hline
\end{tabular}


Serrano, María

Serroni, Scolastica

Settanni, L.

Seubert, John M.

Sextl, G.

Seyfert, Hans-Martin

Seymour, E. Mitchell

Shah, Kavita

Shahgaldian, Patrick

Shahidi, Sheila

Shaik, Ahmad Ali

Shaik, Sason

Shan, Wei-Guang

Shang, Ming-Ying

Shanmugam, K. T.

Shapiro, Nancy L.

Sharma, Dipali

Sharpless, Norman

Shcharbin, Dzmitry

She, Gaimei

Shecterle, Linda M.

Shen, Juan

Shen, Ya-Ching

Shen, Yue-Mao

Sheng, Chunquan

Sherry, A. Dean

Sheu, Jyh-Horng

Shi, Da-Qing

Shi, Fa-Nian

Shi, Gangrong

Shi, Qian

Shi, Xiangyang

Shi, Xiaodong

Shiau, Chung-Wai

Shibasaki, Masakatsu

Shieh, Dar-Bin

Shih, Ming-Der

Shih, Wen-Pin

Shikov, Alexander N.

Shilin, Chen

Shim, Soon-Mi

Shima, Seigo
Shimada, Ichio

Shimada, Tsutomu

Shimizu, Hiroki

Shimoda, Hiroshi

Shin, Hyung Shik

Shin, Sung Chul

Shinitzky, M.

Shipley, Paul

Shirota, Yasuhiko

Shoben, Abigail B.

Short, John D.

Shova, S.

Shoyama, Yukihiro

Shridhar V. Andurkar, Shridhar

Shum, Anderson Ho Cheung

Shuto, Tsuyoshi

Si, Ting

Siddiqi, Rahmanullah

Siddiquee, Tasneem A.

Siebert, Hans-Christian

Sievänen, Elina

Silva, Carlos M.

Silva, Daniel Varon

Silva, Filomena

Silva, Hélder Daniel

Silva, Jefferson Rocha A.

Silván, Jose M.

Silvestri, Romano

Simal-Gándara, Jesús

Simerska, Pavla

Simirgiotis, Mario

Simmonds, Monique S. J.

Simonneaux, Gerard

Simonsen, Henrik

Simpson, Garth

Singh, Ashutosh

Singh, Baljit

Singh, Itender

Singh, S. K.

Singh, Suresh B.

Singh, Udai P.

Siniscalco, Dario
Siodłak, Dawid

Sippl, Wolfgang

Sips, Patrick Y.

Siqueira, Ionara

Sironi, Maurizio

Sissi, Claudia

Sivakumar, Manickam

Skaltsa, Helen D.

Skrobik, Yoanna

Sladic, Dusan

Sławiński, Jarosław

Sliva, Daniel

Smart, Robert C.

Šmejkal, Karel

Smietana, Michael

Smith, Alan

Smith, Robert E.

Smoleński, Piotr

Soares, Luiz

Sobarzo-Sánchez, Eduardo

Sobczak, Izabela

Sobkowski, Michal

Soengas, Raquel

Sokolenko, Taras M.

Solfrizzo, M.

Soliman, Karam F. A.

Song, Baoan

Song, Jinhua

Song, Yang

Song, Yang-Heon

Song, Zhihong

Sooman, Linda

Sørlie, Morten

Soto, Carmen

Soukoulis, Christos

Soulère, Laurent

Sousa, Andreia F.

Sousa, Maria João

Souza, Lauro M.

Sozio, Piera

Sozzi, Gabriella

Spangenberg, Hans Christian 
Sparr, C.

Spatafora, Carmela

Specht, Alexandre

Speer, Karl

Spina, Federica

Spiteller, M.

Sreelatha, S.

Sridhar, Radhakrishnan

Srivatsan, Eri S.

Stadler, Marc

Staerk, Dan

Stafford, Gary

Staines, Henry M.

Stamatatos, Theocharis C.

Stanisz, Beata

Stanković, Milan S.

Stark, Timo D.

Starks, Courtney M.

Steenkamp, Vanessa

Stefani, Hélio A.

Steinbrenner, Holger

Stephanidou-Stephanatou, Julia

Stevanato, Roberto

Stevenson, Philip C.

Stoddart, Fraser

Stojanovic, Ivana

Stolle, Achim

Stone-Elander, Sharon

Stone, Michael

Stonik, Valentin A.

Stoppacher, Norbert

Strano, Michael S.

Stratakis, Manolis

Stratmann, Johannes W.

Struga, Marta

Su, Shu-Jem

Suarez, Alirica

Subramani, Ramesh

Sugimoto, Keiichiro

Sugimura, Hideyuki

Sugumaran, Manickam

Suh, Hyung Joo
Sun, Daekyu

Sun, Haoran

Sun, Hui

Sun, Jingzhi

Sun, Kang

Sun, Ming-Liang

Sun, Peng

Sundberg, Richard

Sung, Ping-Jyun

Surup, Frank

Sussman, Fredy

Sutherland, J. B.

Sutthivaiyakit, Somyote

Suzuki, Hiroyoshi

Suzuki, Nobuaki

Suzuki, Yasuo

Svete, Jurij

Sypecka, Joanna

Szeląg, Małgorzata

Szewczyk, Nathaniel J.

Taatjes, Craig A.

Tabata, Jun

Taglialatela-Scafati, Orazio

Taglietti, Angelo

Tago, Megumi

Tahara, Hidetoshi

Tai, Guihua

Taïeb, David

Taioli, Simone

Takagi, Ryukichi

Takase, Masayoshi

Takasu, Kiyosei

Takeda, Kei

Takeda, Yoshiyu

Takemori, Hiroshi

Taketsugu, Tetsuya

Takimiya, Kazuo

Talbot, Jennifer

Talib, Wamidh H.

Talukdar, Ranajit K.

Tan, Nguan Soon

Tan, Yi
Tanabe, Yoo

Tanaka, Hitoshi

Tanaka, Masakazu

Tang, Chih-Hsin

Tang, Wei

Tang, Li

Tang, Xing

Tang, Yiqun

Tao, Feng

Tao, Lei

Tapia, Alejandro

Tapondjou, La

Tarasiuk, Jolanta

Taupitz, Matthias

Tauskela, Joe

Tava, Aldo

Tegoni, Matteo

Teixeira, Róbson

Tel-Zur, Noemi

Tempesta, S.

Teng, Chieh-Lin

Tepe, Jetze J.

Terasaka, Naoki

Terekhova, Irina V.

Terenzi, Hernán F.

Teschke, Rolf

Tesio, Franco

Testa, Ugo

Tezuka, Yasuhiro

Thevissen, Karin

Thiéry, Valérie

Thomas, Brian F.

Thomas, Christophe M.

Thomas, Eric

Thomas, Raymond $\mathrm{H}$.

Thompson, Colin

Thompson, Kyle J.

Thomson, Regan J.

Timperio, Anna Maria

Titorenko, Vladimir I.

Tittmann, Kai

Tjandra, Nico 
Tokunaga, Makoto

Tolstoy, Pete M.

Tomi, Félix

Tomic, Oliver

Tommonaro, Giuseppina

Tong, Meiping

Tonks, Nick K.

tori, Motoo

Torii, Seiji

Török, Marianna

Toropov, Andrey A.

Torrens, Francisco

Tortorano, Anna Maria

Toshiro, Noshita

Toth, Gabor

Touaibia, Mohamed

Tournier, Nicolas

Touzani, Rachid

Toyooka, Naoki

Tramontano, Enzo

Trehy, Michael L.

Trevisan, Gabriela

Trevitt, Adam

Trindade, Alexandre

Tron, Gian Cesare

Truhlar, Donlad

Tsai, Chia-Wen

Tsai, Li-Chu

Tsai, Shauwei

Tsai, Tung-Hu

Tsai, Yow-Fu

Tsalev, Dimiter

Tsanaktsidis, John

Tsatsaroni, E.

Tsay, Chien-Yie

Tsoleridis, Constantinos A.

Tsopmo, Apollinaire

Tsourkas, Andrew

Tsuboi, Yasuyuki

$\mathrm{Tu}, \mathrm{Tao}$

Tups, Alexander

Turel, Iztok
Tuzimski, Tomasz

Tuziuti, Toru

Tzakos, Andreas G.

Tzen, Jason Tc

Ueda, Kouhei

Ueda, Mitsuhiro

Ullah, Saif

Uquiche, Edgar

Urabe, Hirokazu

Urbanczyk-Lipkowska, Zofia

Usami, Yoshihide

Usenik, VAlentina

Utrilla, Pilar

Vacca, Rosa

Vaidyanathan, Ganesan

Vaira, Dino

Valencia, Gregorio

Valensin, Daniela

Valentao, Patrícia

Valentin, Eulogio

Valentini, Massimiliano

Valero, M.

Valledor, Annabel

Van Bogaert, I. N. A.

Van Dam, Mike

Van De Graaf, Stan F. J.

Van De Venter, Maryna

Van De Vijver, Pieter

Van Den Boorn, Jasper G.

Van Den Elsen, Jean Mh

Van Der Aa, Lieke

Van Dongen, Guus A. M. S.

Van Eijk, Marco

Van Hoek, Monique L.

Van Kaer, Luc

Van Lanen, Steven

Van Nhien, Albert Nguyen

Van Nieuwenhove, Carina P.

Van Ree, Teunis

Van Rij, Ronald,

Van Staden, Van

Van Valckenborgh, Els
Vandamme, Marc

Vanden Eynde, ean Jacques

Vanderlinden, Evelien

Vanetti, Maria Cristina Dantas

Vannacci, Alfredo

Vaquero, M. J. Rodríguez

Varese, Giovanna Cristina

Vargas-Baca, Ingacio

Vargas-Bello-Pérez, E.

Vargas-Rodriguez, Yalma L.

Varney, Veronica A.

Vasconcelos, Vitor

Vasilevich, Natalya I.

Vavř́ḱková, Eva

Vavvas, Demetrios G.

Vayssade, Muriel

Vaz, Maria G. F.

Vedani, Angelo

Vediyappan, Govindsamy

Veitch, Nigel C.

Veleirinho, Beatriz

Velez, Zélia

Velkov, Tony

Vennerstrom, Jonathan

Venturelli, Sascha

Verardo, Vito

Verbruggen, Alfons

Vermerris, Wilfred

Verrax, Julien

Vetter, Ingrid R.

Vetvicka, Vaclav

Viappiani, Cristiano

Vicario, Jose L.

Vicente, Gonzalo

Vicente, José

Vidal, Hilario

Vieira, Ceferina

Vieira, Ivo José Curcino

Vieira, Margarida C.

Vieira, Nirton

Vilas-Boas, Miguel

Vilegas, Wagner 
Villa, Federica

Villegas, Alejandro Madrid

Villmann, Carmen

Vinsova, Jarmila

Virag, Jitka A. I.

Viricelle, J.-P.

Viruel, Maria Angeles

Vitali, Luca

Vitalini, Sara

Vitorović-Todorović, Maja D.

Vivier, Melané A.

Vlashi, Erina

Vlastos, Dimitris

Vock, Carsten

Voituriez, Arnaud

Volf, Petr

Volpatti, Donatella

Volpi, Nicola

von Recum, H. A.

Vosjan, Maria

Vougioukalakis, Georgios C.

Vriens, Joris

Vuorela, Pia

Wadsak, W.

Wagner, Carl E.

Wähälä, Kristiina

Wahlqvist, Mark L.

Wakamatsu, Hideaki

Wakshlag, Joseph

Waksmundzka-Hajnos, Monika

Walsby, Charles J.

Walseth, Timothy F.

Walz, Riger

Wan, Hayley

Wan, Jian-Bo

Wan, Meng-Wei

Wanderley, Almir G.

Wang, Changchun

Wang, Changguan

Wang, Chi Chiu

Wang, Chih Kuang

Wang, Ching-Chiung
Wang, Chun-Ru

Wang, Chung-Yi

Wang, Cunde

Wang, Feng

Wang, Fuyi

Wang, Guowei

Wang, Hao-Yang

Wang, Hexiang

Wang, Hongbing

Wang, Hui-Min David

Wang, Jeffrey

Wang, Jeh-Jeng

Wang, Jian-Wu

Wang, Jigang

Wang, Jinchuang

Wang, Kai-Yun

Wang, Leyong

Wang, Limin

Wang, Meng-Jiy

Wang, Peng George

Wang, Richard Y.

Wang, Shenguang

Wang, Shengyang

Wang, Tzu-Pin

Wang, Wei-Hsien

Wang, Wen

Wang, Wen-Jwu

Wang, Xi Cun

Wang, Xianbao

Wang, Xianzhong

Wang, Xiaowu

Wang, Xinkun

Wang, Xunsi

Wang, Y.

Wang, Yan-Qing

Wang, Ying

Wang, Yingyao

Wang, Yingying

Wang, Yonghua

Wang, Zhanguo

Wang, Zhanzhong

Wang, Zhijun
Waring, Michael

Waser, Mario

Watanabe, Naoharu

Waters, Stephen P.

Wawra, Stephan

Weghe, Pierre Van De

Wei, Jian-He

Wei, Jianhe

Weisman, Gary

Welch, K. D.

Wells, Timothy N. C.

Welsh, William J.

Wertz, Philip

Wesolowski, Marek

West, Caroline

White, Alex

Whitfield, Dennis M.

Wildhalm, Michael

Willars, Gary

Willför, Stefan M.

Williams, David E.

Williams, Ffrancon

Williams, Ian H.

Willner, Itamar

Wills, Martin

Wilson, Lee D.

Wilson, W. David

Wimmer, Zdenek

Windt, Michael

Wingfield, Paul T.

Wingler, Kirstin

Winiarska, Katarzyna

Wondrak, Georg T.

Wong, Chi Chun

Wong, Chi-Huey

Wong, Harvey

Wong, Henry N. C.

Wong, P. K.

Wong, Wai-Yeung

Wong, Wallace

Woo, Mi Hee

Wood, Michael R. 
Wood, W. Gibson

Wood, Warren J. L.

Woods, Mark

Worth, Randall G.

Wrackmeyer, Bernd

Wrenger, Carsten

Wright, Aaron T.

Wright, Dennis

$\mathrm{Wu}$, Alan $\mathrm{Hb}$

$\mathrm{Wu}$, Albert M.

$\mathrm{Wu}$, Bin

$\mathrm{Wu}$, Chieh-Hsi

Wu, Chih-Hsiung

Wu, Chin-Chung

Wu, Christine D.

Wu, Chun-Chi

$\mathrm{Wu}$, Defeng

Wu, Ho-Shing

Wu, Horng Cherng

$\mathrm{Wu}$, Huayue

$\mathrm{Wu}$, Huiquan

Wu, Jie

Wu, Jin-Yi

$\mathrm{Wu}$, Li-Chen

Wu, Ming-Jiuan

$\mathrm{Wu}, \mathrm{Pao}-\mathrm{Chu}$

Wu, Peng

$\mathrm{Wu}$, Qiong

Wu, Shih-Hsiung

Wu, Shihua

Wu, Shu-Jing

Wu, Tian-Xiang

$\mathrm{Wu}$, Tzi-Yi

$\mathrm{Wu}$, Tzong-Yuan

Wu, Xiao-Feng

Wu, Yang-Chang

$\mathrm{Wu}$, Yong

Wu, Zhaoxin

Wuest, Frank

$\mathrm{Xi}$, Chuanwu

Xi, Zhen

Xiang, Lan
Xiao, Dequan

Xiao, Qiang

Xiao, Wei-Lei

Xie, Haihui

Xie, Pei-Shan

Xiong, Bing

Xiong, Fei

Xodo, Luigi E.

$\mathrm{Xu}$, Baoshan

$\mathrm{Xu}$, Changjie

$\mathrm{Xu}$, Chunlin

$\mathrm{Xu}$, Huajian

$\mathrm{Xu}$, Jian-Hong

$\mathrm{Xu}, \mathrm{Li}-$ Wen

$\mathrm{Xu}$, Shichao

$\mathrm{Xu}$, Xudong

$\mathrm{Xu}$, Zhenbo

Xue, Hui

Xue, Ming

Xue, Ying

Yadav, Hariom

Yadav, Madhav

Yaegaki, Ken

Yagi, Kiyohito

Yalcin, D.

Yamada, Hiroko

Yamada, Shizuka

Yamagishi, M.

Yamaguchi, Masahiko

Yamaguchi, Yoshiki

Yamaki, Koji

Yamamoto, Norio

Yamamoto, Yasunori

Yamamoto, Yoshinori

Yamato, Takehiko

Yamauchi, Satoshi

Yamauchi, Takahiro

Yamazaki, Shigekazu

Yamazaki, Takashi

Yanada, Reiko

Yanagita, Ryo C.

Yang, Bao
Yang, Changqing

Yang, Chi-Chiang

Yang, Guangzhong

Yang, Hsin-Ling

Yang, Jian

Yang, Kun-Lin

Yang, Li

Yang, Ling

Yang, Liqun

Yang, Liuqing

Yang, Meihua

Yang, S. C.

Yang, Shun-Fa

Yang, Te-Fang

Yang, Wen-Chin

Yang, Xiao-Hua

Yang, Xichuan

Yang, Ying-Wei

Yang, Yuedong

Yang, Yuh-Shyong

Yang, Z.-Q.

Yang, Zhijun

Yao, Ching Fa

Yao, Guangmin

Yao, Jianhua

Yao, Mei-Cun

Yao, Ping

Yatsunyk, Liliya

Ye, Song

Ye, Xingqian

Ye, Zhengfang

Yen, Tzu-Chen

Yenjai, Chavi

Yeo, Siok-Koon

Yeung, Bryan K. S.

Yeung, Ying-Yeung

Yew, Wen Shan

Yim, Yong-Hyeon

Yli-Kauhaluoma, Jari

Yokoyama, Yoshihito

Yoo, Eun-Sook

Yoo, Hyuk Sand 
Yoon, Do Yeung

Yoon, Do-Young

Yoon, Juyoung

Yoshida, Hiroto

Yoshida, Takashi

Yoshimatsu, Mitsuhiro

Yoshimi, Yasuharu

Youn, Buhyun

$\mathrm{Yu}, \mathrm{Chu}-\mathrm{Yi}$

$\mathrm{Yu}$, Chung-Shan

Yu, Jianmei

Yu, Steve S.-F.

Yu, Wen-Bin

Yuan, C. S.

Yuan, Lujiang

Yuan, Qipeng

Yuan, Weizhong

Yuan, Zhong

Yuanjiang, Pan

Yuasa, Hideya

Yuasa, Junpei

Yuen, John Wm

Yuk, Hyun-Gyun

Yusa, Shin-Ichi

Zabaras, Dimitrios

Zacchetti, Daniele

Zacchino, Susana

Zaharia, Valentin

Zalapa, Juan E.

Zalapa, Juan Ernesto

Zamani, Akram

Zamora-Ros, Raul

Zandi-Sohani, N.

Zangara, Andrea

Zaprutko, Lucjusz

Zarrelli, Armando

Zaug, Joe

Zech, S. G.

Zeng, Aiwu

Zeng, Dexing

Zeng, Minfeng

Zeng, Su
Zhang, Guojian

Zhang, Han-Ting

Zhang, Hanqi

Zhang, Ji-Lin

Zhang, Jianjun

Zhang, Jun

Zhang, Lian-Feng

Zhang, Liangren

Zhang, Lu-Yong

Zhang, Pei-Cheng

Zhang, Pengfei

Zhang, Qinghai

Zhang, Weiyun

Zhang, Wen

Zhang, Wenbing

Zhang, Wenchang

Zhang, Xiao-Mei

Zhang, Xiaokun

Zhang, Xiuli

Zhang, Xiurong

Zhang, Yan-Bo

Zhang, Yang

Zhang, Yansheng

Zhang, Yin

Zhang, Yingjun

Zhang, Yu

Zhao, Chun-Xia

Zhao, Chunshun

Zhao, Haifeng

Zhao, Hong

Zhao, Hua

Zhao, Jianzhang

Zhao, Jing

Zhao, Ming

Zhao, Mouming

Zhao, Peng

Zhao, Qinshi

Zhao, Yongxi

Zhao, youxin

Zheng, Li

Zheng, Qi-Huang

Zheng, Wei
Zhong, Yu-Wu

Zhou, Dong-mei

Zhou, Guangxiong

Zhou, Guohua

Zhou, Hong

Zhou, Huiping

Zhou, Li-Gang

Zhou, Pei

Zhou, Xue-Rong

Zhou, Yifa

Zhu, Duanwei

Zhu, Hai-Liang

Zhu, Hongjun

Zhu, Jianglong

Zhu, Nanwen

Zhu, Qin-Yu

Zhu, Wei-Ming

Zhu, Weihong

Zhu, Ying

Zhuang, Xiuli

Zia, Muhammad

Zielinska, Danuta

Zieliński, Henryk

Zimmerman, Steven C.

Żołnowska, Beata

Zondlo, Neal J.

Zong, M. H.

Zong, Xu-Xiao

Zorc, Branka

Zou, Chenyu

Zou, Yong

Zou, Zhongmei

Zoumpoulakis, Panagiotis

Zrenner, Rita

Kochi, Takuya 
(C) 2014 by the authors; licensee MDPI, Basel, Switzerland. This article is an open access article distributed under the terms and conditions of the Creative Commons Attribution license (http://creativecommons.org/licenses/by/3.0/). 